\title{
Precursor phase of $\mathrm{O}_{2}$ transition
}

\section{Monolayer films of molecular oxygen adsorbed on graphite undergo two phase transitions - a lattice distortion and a magnetic ordering - and this presents calculators with a challenge.}

WHEN is a phase transition to a disorderly state a necessary prelude to a ferromagnetic phase transition involving long-range ordering of the spins in a lattice? The simple answer to this apparently paradoxical question is that the material must be molecular oxygen physically adsorbed in graphite as a two-dimensional layer and at a temperature of $11.5 \mathrm{~K}$ or thereabouts. The case has been sustained by a neat and sensitive measurement of adsorbed oxygen at low temperatures by R. Marx and B. Christoffer of the low-temperature laboratory of the University of Duisburg in West Germany (Phys. Rev. Lett. 51, 790794; 1983).

As a gas or liquid, molecular oxygen is familiarly known to be paramagnetic: the two outermost electrons have parallel spins and the net spin of the system is unity under all known circumstances. Single molecules of oxygen physically adsorbed on a graphite surface lie flat, with both atoms held to the surface, but as the occupancy of surface sites increases, oxygen molecules tend to stand on end. When there are 1.7 molecules of oxygen for each pair of possible adsorption sites on the surface, all of them are standing upright, perpendicular to the surface. The result is an array on a two-dimensional equilateral triangular lattice of molecules each carrying a magnetic moment parallel to the surface (and out of register with the underlying lattice). In this system a long-range ordering of magnetic spins should be possible, as calculations of the properties of two-dimensional Ising lattices have shown.

For several years it has been known that there is indeed a state of long-range magnetic order below about $11.5 \mathrm{~K}$ in which molecular oxygen behaves literally as a two-dimensional antiferromagnet, a consequence of the nature of the electronexchange interaction between oxygen molecules which tends to align neighbouring spins in opposite directions. But as the lattice of the adsorption sites is triangular, it is not possible for the spins to be aligned so that all pairs of neighbouring spins are anti-parallel. Technically, the long-range magnetic ordering is "frustrated".

So how can a monomolecular film of molecular oxygen be a two-dimensional antiferromagnet? Only by some distortion of the two-dimensional lattice that removes the frustrating geometrical symmetry of the underlying equilateral triangular (or centred hexagonal) lattice. That there is, indeed, such a distortion has been shown by neutron diffraction of molecular oxygen films (McTague, J.P. and Nielsen, $M$. Phys. Rev. B19, 3096; 1979). It consists of a distortion of the basic equilateral triangle (with sides of $3.27 \AA$ ) into an isosceles triangle in which one side is lengthened (to $3.43 \AA$ ) and the other two shortened (to $3.20 \AA)$. In such a distorted twodimensional array of oxygen molecules, the perfectly ordered antiferromagnetic state is that in which all spins point along the directions of the lengthened dimensions (in one sense or the opposite), and in which the rows of sites along the shortened dimensions of the lattice are occupied consecutively by alternating spins. Thus the exchange energy between neighbouring sites is a true minimum, and the net magnetic moment is zero, so producing an antiferromagnetic phase. The question that Marx and Christoffer tackle is whether the phase transitions consisting of lattice distortion and magnetic ordering occur together or separately.

They have been able to measure the specific heat of oxygen adsorbed on graphite sensitively enough to show that there are indeed two phase transitions, at temperatures of $11.55 \mathrm{~K}$ and $11.32 \mathrm{~K}$ respectively. Part of the trick is to couple together two adsorption cells, one containing foil graphite "rolled like a carpet" and the other a stack of graphite sheets so as to obtain the specific heat of the adsorbed oxygen in fields which are either parallel or perpendicular to the graphite surface. The results (open circles in the diagram) show that when there is no field, there are indeed two distinct peaks in the plot of specific heat against temperature and, so, two successive phase transitions - not one.

Because the antiferromagnetic state is unattainable in the strictly symmetric triangular lattice, it is natural to infer that lattice distortion precedes the antiferromagnetic transition as the temperature falls. Marx and Christoffer have, however, proved the point by showing that the second transition is virtually abolished in a field of $1.1 \mathrm{~T}$ parallel to the adsorption surfaces (filled circles in the diagram) but not by a field perpendicular to its surface.

Such behaviour has previously been described only in three dimensions. Thus the material $\mathrm{Dy} \mathrm{VO}_{4}$, antiferromagnetic at low temperatures, has two phase transitions at $3 \mathrm{~K}$ and $14 \mathrm{~K}$ which were interpreted as magnetic ordering and lattice deformation respectively (Sivardière, J. and Blume, $\mathbf{M}$.
Phys.Rev. B5, 1126-1134; 1972).

But how can a material which has never previously been near liquid helium "know" that its lattice must distort at some critical temperature to make possible antiferromagnetism at some lower temperature? Anthropomorphism is uncalled for, as the interaction of the magnetic spins

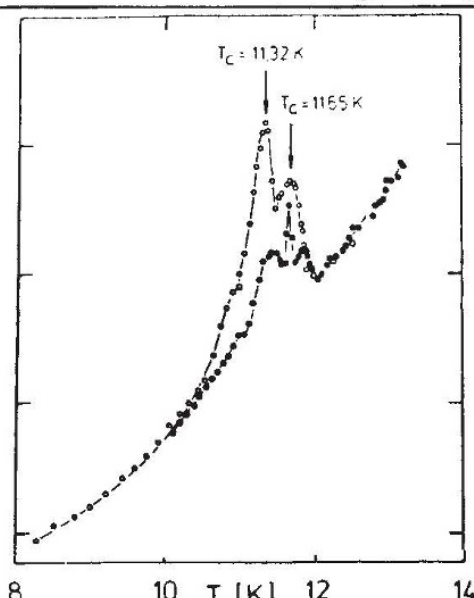

Specific heat per molecule (arbitrary units) for a monolayer of $\mathrm{O}_{2}$ molecules on graphite. Open circles - no magnetic field. Filled circles - 1.1 T field parallel to surface.

must be in part a determinant of the first preparative phase transition; their existence and configuration embody the "knowledge", of the system's low temperature fate.

Unfortunately, even the data Marx and Christoffer have produced on the specific heat of monolayer oxygen are not accurate enough to demonstrate that influence quantitatively. The authors do, however, say that the variation of specific heat with temperature in differently oriented magnetic fields shows that the lower transition is an Ising transition (order-disorder on a two-dimensional lattice occupied by two-valued scalar spins interacting only with nearest neighbours) and that the higher transition is a Potts transition (an elaboration of the Ising lattice.)

What this implies is that the properties of oxygen adsorbed on graphite should be almost exactly calculable, at least in the bottom left-hand (low temperature, low occupancy) corner of the phase diagram. Awschalom et al. have suggested (Phys. Rev.Lett. 51, 586; 1983) that the rest of the phase diagram shows that when two regular layers have been filled, further adsorbed oxygen has the constitution of the bulk phases. This may make the challenge irresistible.

John Maddox 